\title{
Flora do Rio de Janeiro: Myristicaceae
}

\author{
Flora of Rio de Janeiro: Myristicaceae
}

\author{
Lanúzia Grandini Quintanilha ${ }^{1} \&$ Adriana Quintella Lobão ${ }^{1}$
}

\begin{abstract}
Resumo
Myristicaceae inclui cerca de 20 gêneros e 500 espécies e no Brasil ocorrem cinco gêneros e 64 espécies, sendo 59 na Amazônia. Virola é o gênero mais amplamente distribuído no Brasil. No Rio de Janeiro, encontra-se apenas o gênero Virola com duas espécies, Virola bicuhyba e V. gardneri. Estas duas espécies são encontradas na costa sul e centro-norte fluminense. Chave, descrições, comentários taxonômicos, informação sobre fenologia, distribuição geográfica, habitáts de ocorrência, status de conservação e ilustrações são apresentados.
\end{abstract}

Palavras-chave: Virola, Floresta Atlântica, angiospermas basais.

\begin{abstract}
Myristicaceae includes about 20 genera and 500 species and in Brazil there are five genera and 64 species, 59 in the Amazon. Virola is the genus most widely distributed in Brazil. In Rio de Janeiro, the only two species found are Virola bicuhyba and V. gardneri. These two species are seen on the south coast and north-central part of the state. Key, descriptions, taxonomic comments, information on phenology, geographic distribution, habitats, conservation status and illustrations are presented.
\end{abstract}

Key words: Virola, Atlantic Forest, basal angiosperms.

\section{Myristicaceae}

Frequentemente árvores, casca interna com exsudado avermelhado ou que em contato com ar oxida para o vermelho. Folhas simples, alternas, sem estípulas. Inflorescência fasciculado-racemosa ou paniculada; flores diminutas, incospícuas, dioicas, raramente monóica, actinomorfas, monoclamídeas, trímeras, tépalas, parcialmente conatas; estames 2-numerosos, monadelfas; ovário súpero, unicarpelar, placentação basal, uniovulado. Fruto baciforme descente por deiscência ventral e dorsal, carnosa ou sublenhosa, semente envolta por arilo carnoso, inteiro ou fendido irregularmente (Caruso \& Cordeiro 2000; Rodrigues 1997; Vicentini \& Rodrigues 1999).

Myristicaceae é uma família pertencente à Magnoliales (APGIV 2016). Na América ela ocorre desde o sul do México até Bolívia e parte setentrional do Rio Grande do Sul, no Brasil (Belota Filho 1994). A família inclui cerca de 20 gêneros e 500 espécies e no Brasil ocorrem cinco gêneros e 64 espécies, sendo 59 na Amazônia (Souza \& Lorenzi 2008; BFG 2015). Virola é o gênero mais amplamente distribuído no Brasil assim como maior número de espécies (Smith \& Woodhouse 1937). No Rio de Janeiro, encontra-se apenas o gênero Virola com duas espécies, Virola bicuhyba (Schott) Warb. e V. gardneri (A.DC.) Warb. (Rodrigues 1980, 2015). Virola sebifera Aubl. apesar de citada para o Rio de Janeiro (Rodrigues 2015), não será aqui tratada uma vez que o único espécime registrado para o estado é um indivíduo cultivado que não foi encontrado no herbário NY, onde estaria depositado (Glaziou 10030, NY). Virola bicuhyba e $V$. gardneri são encontradas na costa sul e centro-norte fluminense.

\footnotetext{
${ }^{1}$ Universidade Federal Fluminense, Depto. Biologia Geral, Inst. Biologia, R. Outeiro de São João Batista s/n, Campus do Valonguinho, 24020-150, Niterói, RJ, Brasil.

${ }^{2}$ Autor para correspondência: alobao@hotmail.com
} 
1. Virola Aubl. Hist. Pl. Guiane 904. 1775. Myristica L.sens. lat. Swartz, Prod.: 96. 1788.

Árvores. Folhas dísticas. Inflorescência paniculada ou racemiforme; axilar. Flores dioicas, tépalas 3-4(5). Flor masculina com 3 estames, andróforo presente, anteras geralmente conatas até o ápice ou distalmente divergentes. Flor feminina maior que a masculina; estigma geralmente bífido, estilete frequentemente curto ou nulo, ovário densamente tomentoso. Fruto globoso ou elipsoide. Semente globosa ou elíptica (Rodrigues 1980).
Gênero neotropical, no Brasil possui distribuição em Roraima, Amapá, Pará, Amazonas, Tocantins, Acre, Rondônia, Maranhão, Piauí, Ceará, Pernambuco, Bahia, Alagoas, Mato Grosso, Goiás, Distrito Federal, Minas Gerais, Espírito Santo, São Paulo, Paraná, Santa Catarina, Rio Grande do Sul (BFG 2015). No estado do Rio de Janeiro é encontrado na costa sul e centro-norte na Mata Atlântica em solos ricos com elevado teor de umidade e frequentemente elevada altitude (Moreno et al. 2003).

As espécies aqui tratadas para o estado do Rio de Janeiro são V. bicuhyba e Virola gardneri.

\section{Chave de identificação das espécies}

1. Folha estreitamente elíptica, base levemente atenuada, nervuras secundárias 20-30 pares; fruto elipsoide 1. Virola bicuhyba

1'. Folha elíptica a obovada, base fortemente atenuada, nervuras secundárias 13-18 pares; fruto largoelipsoide 2. Virola gardneri

1. Virola bicuhyba (Schott) Warb., Ber. Pharm. Ges.: 225, 1892. Myristica bicuhyba Schott, in C. Sprengel, Syst. Veg. 4(2): 409. 1827.

Fig. 1a-f

Árvore, 8-28 m de alt.; tricomas estrelados. Ramos estriados, glabros a puberulentos. Pecíolo caniculado, glabro a puberulento, 3-18 mm compr. Lâminas foliares 10,4-24,3 × 1,8-5,6 cm, coriáceas, glabrescentes, estreitamente elípticas, ápice agudo a acuminado, base levemente atenuada, ambas as faces puberulentas, nervuras secundárias 20-30 pares. Inflorescência panícula congesta em ambos os sexos. Flor masculina com pedicelo ca. $5 \mathrm{~mm}$ compr.; tépala ca. $2 \times 1 \mathrm{~mm}$; andróforo ca. 1 $\mathrm{mm}$ compr., anteras ca. $1 \mathrm{~mm}$ compr. Flor feminina com pedicelo ca. $3 \mathrm{~mm}$ compr.; tépala ca. $3 \times 3 \mathrm{~mm}$; ovário ca. $2 \times 1 \mathrm{~mm}$. Fruto 2,5-3,3 × 1,7-1,9cm, elipsoide. Semente 2,5-2,3 × 1,5-1,7 cm.

Virola bicuhyba possui distribuição na Bahia, Minas Gerais, Espírito Santo, Rio de Janeiro, São Paulo, Paraná, Santa Catarina e Rio Grande do Sul (Rodrigues 2015). No Rio de Janeiro ocorre regularmente na Floresta Ombrófila. Segundo Rodrigues (1980), a maior altitude até então registrada de ocorrência da espécie foi 780 m.s.m, no Parque Nacional de Itatiaia, Rio de Janeiro. Planta semidecídua, heliófila. Floresce de novembro a abril e agosto e frutifica em junho, julho, setembro e outubro.

Material examinado selecionado: Cachoeiras de Macacu, estrada entre Funchal e Guapiaçu, perto de
Regua, 12.II.2008, fl., O. Thier 1169 (RB); Guapimirim, Estação Ecológica Estadual do Paraiso perto do Centro de Primatologia, 23.IX.2010, st., O. Thier 2022 (RB); Itatiaia, Parque Nacional do Itatiaia, Lote 15, 1.X.1940, fr., W.D. Barros 43 (RB); Nova Iguaçu, arredores da represa do Barrelão, 13.XII.2001, fl., I.M. Silva 472 (RB); Rio das Ostras, Reserva Biológica União, 19.II.1998, fl., P.P. Oliveira 945 (ESA); Santa Maria Madalena, Parque Estadual do Desengano, Sossego, Fazenda Forquinha, próximo ao Rio do Norte, 30.VI.1987, fr., H.C. Lima 3050 (RB); Rio de Janeiro, Vista Chinesa, 22.I.1934, fl., P. Rosa 142 (RB); Silva Jardim, Ilha dos Barbados, 13.IV.1995, fl., P.R.C. Farág 22 (RB).

Virola bicuhyba é conhecida como Bicuiba (Kurtz \& Araujo 2000), Bicuíba Sul, Bicuíbabranca, Bicuuba e Ocuíba (Rodrigues 2015).

A espécie apresenta madeira moderadamente pesada, resistente ao apodrecimento dentro da água e intensamente atacada por insetos xilófagos (Rodrigues 1980) e é empregada na construção civil, carpintaria e marcenaria.

Virola bicuhyba foi tratada em Rodrigues (1980) como Virola oleifera Aubl., entretanto posteriormente Rodrigues (1998) reabilita Virola bicuhyba uma vez que Virola oleifera é um nome inválido.

Virola bicuhyba diferencia-se de $V$. gardneri pelas lâminas foliares estreitamente elípticas e fruto elipsoide em Virola bicuhyba e lâminas foliares elípticas ou obovadas, fortemente atenuadas na base e fruto largo-elipsoide em $V$. gardneri. 


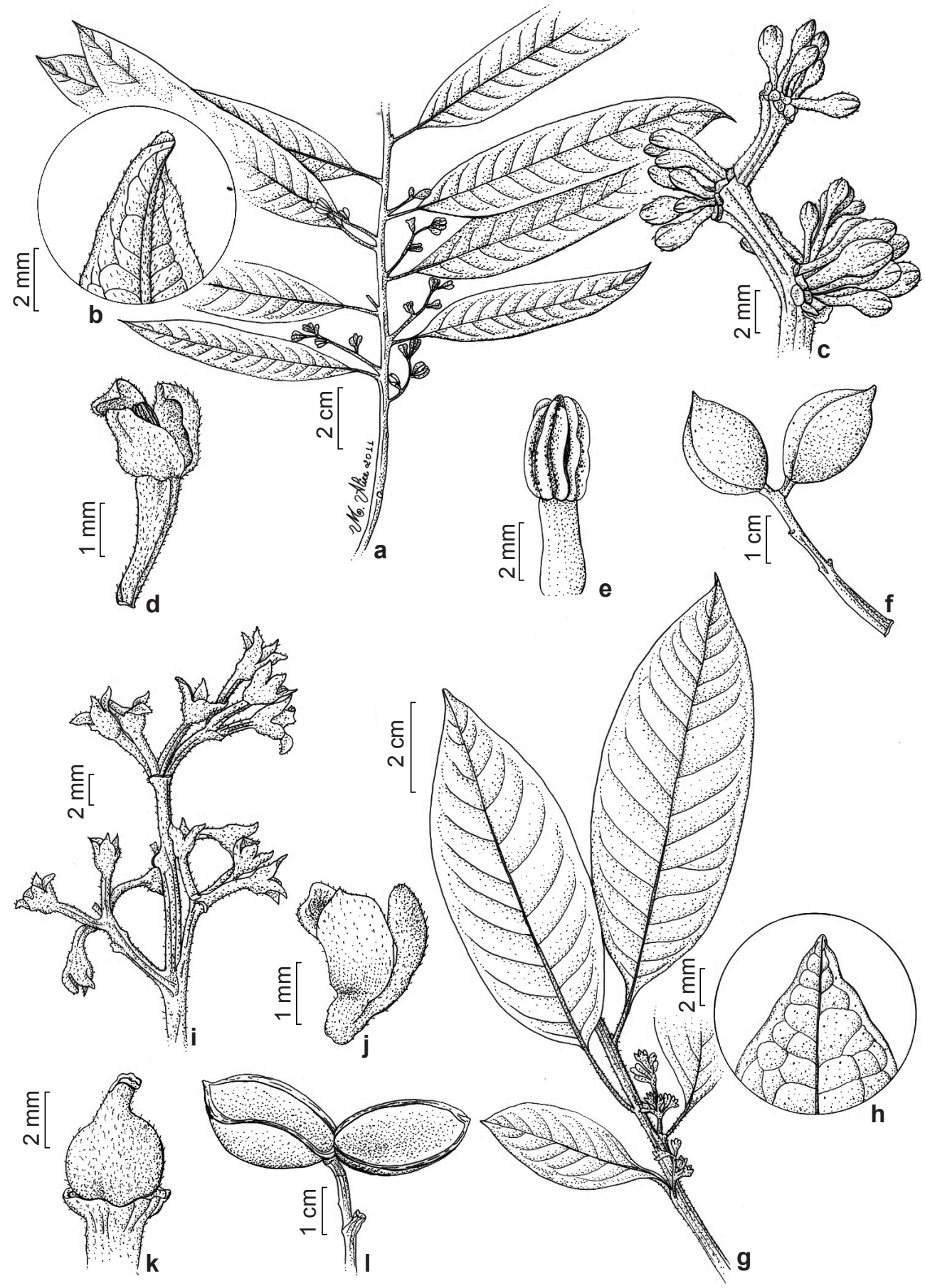

Figura 1 - a-f. Virola bicuhyba - a. ramo com inflorescência; b. detalhe da folha evidenciando indumento; c. parte da inflorescência com flores em botão; d. flor masculina em antese; e. andróforo; f. ramo com frutos imaturos. g-1. Virola gardneri - g. ramo com inflorescência; h. detalhe da face abaxial da folha; i. inflorescência feminina; j. flor feminina; k. ovário; 1. fruto maduro em deiscência apresentando somente o pericarpo (a,b,e. Kurtz 256; c,d. Lage s.n. (RB 120358); f. Lima s.n. (RB 404387); g,h. Ducke s.n. (RB 25105); i,j,k. Barros 559; 1. Farág 175).

Figure 1 - a-f. Virola bicuhyba - a. branch with inflorescence; b. leaf detail showing trichomes; c. part of the inflorescence with flower buds; d. male flower in antesis; e. androforo; f. branch with immature fruits. g-1. Virola gardneri - g. branch with inflorescence; h. detail of the abaxial surface of the leaf; i. female inflorescence; j. female flower; k. ovary; 1. mature fruit opened showing only the pericarp (a,b,e. Kurtz 256; c,d. Lage s.n. (RB 120358); f. Lima s.n. (RB 404387); g,h. Ducke s.n. (RB 25105); i,j,k. Barros 559; 1. Farág 175). 
2. Virola gardneri (A.DC.) Warb., Nova Acta Acad. Caes. Leop. -Carol. Nat. Cur. 68: 192, tab. 6. 1897. Myristica gardneri A.DC. Prodr. Syst. Nat. Reg. Veg. 14(1): 197. 1856.

Fig. 1g-1

Árvore, 10-40 m alt.; tricomas estrelados. Ramos estriados, geralmente glabros a raramente puberulentos. Pecíolo caniculado, glabro a puberulento, 7-20 $\mathrm{mm}$ compr. Lâminas foliares $9,5-17,7 \times 2-7,1 \mathrm{~cm}$, elípticas ou obovadas, levemente coriáceas; ápice acuminado; base fortemente atenuada; face adaxial glabrescente e lustrosa, face abaxial puberulenta; nervuras secundárias 13-18 pares. Inflorescência panícula congesta em ambos os sexos. Flor masculina com pedicelo 3-5 $\mathrm{mm}$; tépalas ca. $2 \times 1 \mathrm{~mm}$; andróforo ca. $1 \mathrm{~mm}$ compr., anteras ca. $0,5 \mathrm{~mm}$ compr. Flor feminina com pedicelo ca. $3 \mathrm{~mm}$ compr.; tépalas ca. $3 \times 1,5 \mathrm{~mm}$; ovário ca. $2 \times 1 \mathrm{~mm}$. Fruto 2,5-3 $\times 2,4-2,9 \mathrm{~cm}$, largo-elipsoide. Semente 2-2,5 $\times$ $1,5-1,8 \mathrm{~cm}$.

Virola gardneri ocorre em Pernambuco, Bahia, Alagoas, Minas Gerais, Espírito Santo, Rio de Janeiro e São Paulo (Rodrigues 2015). No Rio de Janeiro é encontrada em Floresta Ombrófila. A espécie está representada geralmente por árvores de grande porte (Magnanini \& Magnanini 2002). Floresce em janeiro e abril e frutifica em agosto e dezembro.

Material examinado selecionado: Cachoeiras de Macacu, Estação Ecológica Estadual do Paraiso, s/d, st., B.C. Kurtz (RB 227054); Guapimirim, Estação Ecológica Estadual do Paraíso Centro de Primatologia do Rio de Janeiro, st., 6.XI.1984, G. Martinelli 10127 (RB); Itatiaia, Parque Nacional do Itatiaia, lote 15, 19.I.1942, fl., W.D. Barros 559 (RB); Magé, Estação Ecológica Estadual de Paraíso. Centro de Primatológia do RJ. III $^{\circ}$ Distrito, mata secundária entre o Centro de Primatológia e a represa. 5.XII.1984, st., G. Martinelli 10570 (RB); Niterói, Engenho do Mato, Parque Estadual da Serra da Tiririca, Morro do Cordovil, Vale das Borboletas, trilha da Jararaca, ind. 51 do transecto TJ4, st., 31.III.2007, A.A.M. Barros 3016 (RB); Nova Friburgo, estrada entre Hotel Fazenda São João, próximo a porteira do Sítio Baccus, fl., 16.I.1991, S.V.A. Pessoa 545 (RB); Nova Iguaçu, Barrelão, 4.XII.1995, fr., P.R.C. Farág 175 (RB); Silva Jardim, Ilha dos Barbados, 13.IV.1995, fl., P.R.C. Farág 19 (RB); Rio de Janeiro, Jardim Botânico, subindo pela trilha atrás da caixa d'água em direção a Pedra do Marinheiro, fr., 4.VIII.1993, R. Marquete 1166 (RB); Volta Redonda, Floresta da Cicuta, margem do Rio da Água Fria, 17.XI.2001, st., G.R. Souza 2289 (UPCB).

A espécie é conhecida como Bicuibuçu (Rodrigues 2015; Kurtz \& Araujo 2000), Bicuíbaaçu, Bicuíba-da-folha-larga e Bocuva-fêmea (Rodrigues 2015), Bicuíba-vermelha, Mucuhyba,
Pau-sangue, Urucuba (Rodrigues 1997). Há também o registro de Bicuiba Vermelha (Nunes 22534 (NY)). Segundo Rodrigues (1980), a madeira é empregada na confecção de canoas, brinquedos, artefatos de madeira e construção civil, além do óleo da semente servir para fabricação de velas e iluminação; o extrato da amêndoa possui, comprovadamente, atividade antibacteriana contra germes grampositivos e germes ácido-resistentes; a manteiga ou óleo é empregado em fricções das doenças de pele e se a amêndoa for cozida retira mau hálito e conforta estômago. Ainda segundo o autor, a resina que exsuda da casca da árvore ferida aplicada sobre ferimentos é anti-hemorrágica e cicatrizante.

\section{Agradecimentos}

Adriana Q. Lobão agradece à Fundação de Amparo à Pesquisa do estado do Rio de Janeiro (FAPERJ), o apoio financeiro. Lanuzia Quintanilha agradece ao Programa Institucional de bolsas de Iniciação Científica da Pró-Reitoria de Pesquisas, Pós-graduação e Inovação da Universidade Federal Fluminense (PROPPI/UFF), a bolsa concedida.

\section{Referências}

APG (Angiosperm Phylogenetic Group) IV. 2016. An update of the angiosperm phylogenetic group classification for the orders and families of flowering plants: APG IV. Botanical Journal of the Linnean Society 181: 1-20.

Belota Filho, W.L. 1994. As espécies de Iryanthera Warburg (Myristicaceae) da Reserva Florestal Ducke. Dissertação de Mestrado. INPA/FUA, Manaus. 94p.

BFG 2015. Growing knowledge: an overview of seed plant diversity in Brazil. Rodriguésia 66: 1085-1113.

Caruso, M.B.R. \& Cordeiro, I. 2000. Myristicaceae. In: Fiusa de Melo, M.M.R.; Barros, F.; Chiea, S.A.C.; Kirizawa, M.; Jung-Mendaçolli \& Wanderley, M.G.L. (eds.). Vol. 2. Flora fanerogâmica da Ilha do Cardoso. Instituto de Botânica, São Paulo. Pp. 99-103.

Kurtz, B.C. \& Araujo, D.S.D. 2000. Composição florística e estrutura do componente arbóreo de um trecho de Mata Atlântica na Estação Ecológica Estadual do Paraíso, Cachoeiras de Macacu, Rio de Janeiro. Rodriguésia 51: 69-112.

Magnanini, A. \& Magnanini, C. 2002. Árvores gigantescas da terra e as maiores assinaladas no Brasil. CNRBMA, São Paulo. 20p.

Moreno, M.R.; Nascimento, M.T. \& Kurtz, B.C. 2003. Estrutura e composição florística do estrato arbóreo em duas zonas altitudinais na mata atlântica de encosta da região do Imbé, RJ. Acta Botanica Brasilica 17: 371-386. 
Rodrigues, W.A. 1980. Revisão taxonômica das espécies de Virola Aublet (Myristicaceae) do Brasil. Acta Amazônica 10(supl. 1): 1-127.

Rodrigues, W.A. 1997. Myristicaceae. In: Marques, M.C.M.; Vaz, A.S.F. \& Marquete, R. (eds.). Flórula da APA Cairuçu, Parati, RJ: espécies vasculares. Instituto de Pesquisas Jardim Botânico do Rio de Janeiro, Rio de Janeiro. Pp. 310-313.

Rodrigues, W.A. 1998. Reabilitação nomenclatural e taxonômica de Virola bicuhyba (Schott) Warb. (Myristicaceae). Acta Botânica Brasílica 12: 249-252.
Smith, A.C. \& Woodhouse, R.P. 1937. The American species of Myristicaceae. Brittonia 2: 393-510.

Souza, C.V. \& Lorenzi, H. 2008. Botânica sistemática: guia ilustrado para identificação das famílias de angiospermas da flora brasileira, baseado em APG II. Instituto Plantarum, Nova Odessa. 704p.

Vicentini, A. \& Rodrigues, W.A. 1999. Myristicaceae. In: Ribeiro, J.E.L.S. et al. (eds.). Flora da Reserva Ducke. Guia de identificação das plantas vasculares de uma floresta de terra-firme na Amazônia Central. INPA, Manaus. Pp. 136-145.

\section{Lista de exsicatas}

Araujo, R.C. 13 (2). Christo, A.G. 273 (2), 430 (1). Barros, W.D. 43 (1), 559 (2), 607 (1), 3016 (2). Baumgratz, J.F.A. s.n. (2). Borges, R. 45 (1). Ducke, A. 32 (1), s.n. (R 61018) (1). Farág, P.R.C. 19 (2), 22 (1), 160 (1), 175 (2), 176 (1). Farias, D.S. 229 (2), 363 (2). Freire Alemão, F. 372 (1). Gardner, G. 5596 (2). Goes, O.C. 919 (1). Kuhlmann, J.G. 630 (1), 629 (2), 628 (2). Kurtz, B.C. 232 (1), 256 (1), s.n. (2). Lage, C. 142 (1). Lima, H.C. 2287 (1), 2353 (2), 2376 (1), 3050 (1). Luchiari, C. 746 (1). Marquete, R. 1166 (2). Martinelli, G. 9932 (1), 9957 (1), 10021 (2), 10127 (2), 10130 (1), 10148 (2), 10167 (2), 10190 (2), 10214 (2), 10570 (2), 10608 (2). Nadruz, M. 8 (1). Nunes, G.M. 57 (1). Occhioni, P. 247 (1). Pessoa, S.V.A. 545 (2). Silva, I.M. 472 (1). Sobrinho, F.A. 28 (2). Solórzano, A. 73 (1). Sonkin, L. 276 (1). Spolidoro, L.C.V. 9 (1). Sylvestre, L. 788 (1). Velloso, H. s.n. (1). 